\title{
Poincaré on logic and foundations
}

\author{
Gabriele Lolli
}

Published online: 22 May 2013

(C) Centro P.RI.ST.EM, Università Commerciale Luigi Bocconi 2013

\begin{abstract}
Poincaré's intervention in the philosophical debate on mathematics of the beginning of the twentieth century is circumscribed but had lasting effects. The important aspects considered here are his defence of intuition against all forms of logicism and his fierce condemnation of the use of symbolic languages; the criticism of the vicious circle as responsible for all the antinomies, and the influence both on Russell, who was led to adopt the theory of types, and on Weyl, who tried a predicativistic reconstruction of mathematical analysis; the fight with Hilbert for the circular use of induction of the proof of the consistency of arithmetic; his discussions with Peano and Zermelo on the Cantor-Bernstein theorem and on predicative proofs in general.
\end{abstract}

Keywords Henri Poincaré · Mathematical logic . Foundations of mathematics - Antinomies - Pasigraphy · Impredicative definitions - Cantor-Bernstein theorem . Ernst Zermelo · Bertrand Russell · Hermann Weyl · Giuseppe Peano

\section{Introduction}

Henri Poincaré (1854-1912) intervened quite fleetingly in the heated debate about the foundations of mathematics at the beginning of the twentieth century, with not much more than his 1905-06 paper "Les mathématiques et la logique", but he left a crucial and lasting legacy. He persuaded Bertrand Russell (1872-1970) of the responsibility of

G. Lolli ( $₫)$

Scuola Normale Superiore, Piazza dei Cavalieri 7,

56100 Pisa, Italy

e-mail: gabriele.lolli@sns.it impredicative definitions as the source of all antinomies, driving him to the kind of solution he adopted with the theory of types in his Principia mathematica; he influenced Hermann Weyl (1885-1955) towards attempting the construction of a predicativist analysis, giving birth to the subsequent school of predicativism; he spurred the search for new and clearer proofs of the Cantor-Bernstein theorem; he was a thorn in the flesh for David Hilbert's (1862-1943) programme, criticizing the use of induction in metamathematics and so forcing Hilbert to a continuous revision of the notion of finitist methods.

Poincaré's motives lay in a philosophical interest: a defence of his point of view - which he saw as a Kantian one - against the attacks from Leibnizian logicists, and a proof of the necessity of intuition in mathematical knowledge. He was not directly involved in the issues surrounding set theory, but once he entered the arena he could not, or would not, avoid giving his opinion about every controversial theme being debated in the suddenly heated exchange of opinions.

The appearance of [32] divulged the existence of antinomies ${ }^{1}$; they were already known to Georg Cantor (1845-1918) and Ernst Zermelo (1871-1953), and discussed in Hilbert's entourage, inducing Hilbert to press for a solution and to commit himself personally to finding it.

The year 1904, in particular, was full of resounding events. First Julius König (1849-1913), during the international mathematical congress in Heidelberg, announced his proof that the continuum cannot possibly have as its cardinality an aleph, that is, it is not well ordered. The excitement over this announcement died down

\footnotetext{
${ }^{1}$ Russell called them "contradictions" or "paradoxes"; Poincaré appears to have been the first one to use the term "antinomies", in [23, p. 824]; see also [24, p. 154].
} 
immediately, in a day (just the time for Cantor to nearly die of fright), when Zermelo noticed that the proof relied on an erroneous, overly general interpretation of an identity in cardinal arithmetic due to Felix Bernstein (1878-1956). ${ }^{2}$

During the same meeting, Hilbert had claimed that "the views and the research methods most used in logic, as it is traditionally considered, are not up to the requirements of rigour imposed by set theory". The year before, while corresponding with Gottlob Frege (1848-1925), he had been more precise: "the crucial flaw of traditional logic was in assuming that a notion is determined whenever for every entity it is determined whether it lies within the notion or not". In fact, "the point is to ascertain the consistency of the axioms defining the concept" ([11], Hilbert's letter dated 7 November 1903).

In Heidelberg, Hilbert had suggested a way to attack the problem of proving the consistency of arithmetic while avoiding "reliance on another fundamental theory": a gradual, axiomatic introduction of mathematical concepts and a proof of consistency. He had stated that "in order to avoid paradoxes it is necessary to develop, at least in part simultaneously, the laws of logic and arithmetic". He had next considered a toy model of arithmetic based solely on composing and decomposing sequences of objects (basically, a theory of equality between strings of symbols) and proven by an inductive argument that no finite deduction could end in a contradiction.

Two months after the Heidelberg congress, Zermelo sent Hilbert a letter to be published in Mathematische Annalen, in which he proved the well-ordering theorem: every set can be well-ordered [41]. The proof relied on an assumption that in Zermelo's opinion was a "logical principle", and that would later be called "axiom of choice" (or, initially, Zermelo's postulate):

This proof depends on the assumption that there are in general coverings $\gamma$, that is, on the principle that even for an infinite family of sets there always exist correlations by which to every set one of its elements is made to correspond or, formally, that the product of an infinite family of sets, each of them containing at least an element, is different from zero [i.e., the empty set]. Indeed, this logical principle cannot be reduced to a simpler one, but is unwittingly used in many mathematical arguments.

Zermelo's result had ignited one of the most heated debates ever to take place between mathematicians (with the participation of representatives from all countries: France, Italy, England, Germany, and United States), both

\footnotetext{
$\overline{2}$ According to a different reconstruction, based on the statements of those present, the correction arrived later, in a 1904 paper by Felix Hausdorff (1868-1942). See [9, p. 312].
}

on the axiom of choice and on other details of the proof and on the set-theoretical antinomies. In the meantime, new antinomies of a linguistic type had appeared, beginning with [30].

In 1908 Zermelo, while advancing a new proof of his theorem, exhibited a confutation of all criticism sustained by the first one, recapitulating those years' debates, including those in which Poincaré had a leading role. Almost as if to close a period of disorientation, in 1908 both the axiomatization of set theory in [43] and the formulation of the theory of types in [35] were published.

But when Poincaré wrote "Les mathématiques et la logique", the main object of his criticism were [4] and [5], and his role seemed marginal with respect to the ongoing debate (for instance, as we shall see, he took no position about the axiom of choice); further, he was influenced by the peculiar and questionable synthesis made by Louis Couturat (1868-1914). The term "logicistes" as used by Poincaré was not common, but rather strongly idiosyncratic: this same label was applied to Peano and pasigraphy, Cantorism and Russell.

\section{The philosophy of Poincaré}

The view of Poincaré which must be kept in mind in order to understand his contribution to the debate is clearly expressed in La science et l'hypothèse:

The very possibility of mathematical science seems an insoluble contradiction. If this science is deductive outwardly only, from whence is derived that perfect rigour which is challenged by none? If, on the contrary, all the propositions which it enunciates may be derived in order by the rules of formal logic, how is it that mathematics is not reduced to a gigantic tautology? The syllogism can teach us nothing essentially new, and if everything must spring from the principle of identity, then everything should be capable of being reduced to that principle. Are we then to admit that the enunciations of all the theorems with which so many volumes are filled, are only indirect ways of saying that $A$ is $A$ ? ([22], p. 1$){ }^{3}$

In order to solve the contradiction, it is not enough to observe that the axioms are not logical truths.

No doubt we may refer back to axioms which are at the source of all these reasonings. If it is felt that they cannot be reduced to the principle of contradiction, if we decline to see in them any more than experimental

\footnotetext{
3 This and all other translations from Science and Hypothesis are taken from the 1905 translation by W.J. Greenstreet.
} 
facts which have no part or lot in mathematical necessity, there is still one resource left to us: we may class them among a priori synthetic views. But this is no solution of the difficulty - it is merely giving it a name; and even if the nature of the synthetic views had no longer for us any mystery, the contradiction would not have disappeared; it would have only been shirked. Syllogistic reasoning remains incapable of adding anything to the data that are given it; the data are reduced to axioms, and that is all we should find in the conclusions ([22], p. 10).

If all the inferences used in mathematics were just logical ones,

No theorem can be new unless a new axiom intervenes in its demonstration; reasoning can only give us immediately evident truths borrowed from direct intuition; it would only be an intermediary parasite. Should we not therefore have reason for asking if the syllogistic apparatus serves only to disguise what we have borrowed? ([22], p. 2).

But in Poincaré's opinion it is beyond dispute that mathematical reasoning can give us new knowledge and usually does. Mathematics has a kind of creative power. The solution to the ostensible contradiction amounts to recognizing that mathematical reasoning is based on specific principles, which Poincaré calls a priori synthetic ones, probably for want of a better term. From the quotes above it is understood that here "synthetic" means "that which increases knowledge", and "a priori" "that which has a necessity independent of experience". Terminology and outward explicit defence of Kant notwithstanding, Kantian philosophy is completely absent here; when Poincaré mentions intuition, he does not refer to senses. In [21] he explicitly distinguished intuition from imagination and senses. He had also claimed that intuition does not simply play a role in the initial principles, but is necessary to understand a proof, even after the correctness of every step has been verified. ${ }^{4}$

A mathematical truth is given by intuition if we recognize it to be true without having or feeling the need for a proof; a principle is a priori synthetic if it is not a logical one and if it gives mathematical knowledge, as is the case for induction.

\section{Against logicists}

According to logicists, it was possible to reduce mathematics to logic without resorting to any particular principle.

\footnotetext{
4 This interpretation of Poincaré's a priori synthetic is developed in [8]. In [12], however, Goldfarb emphasizes Poincaré's attention for psychological aspects, in the debate with logicists.
}

In the revision of [23] published in Science et méthode (1908), we read that this thesis follows from the work of Cantor in which he introduced actual infinity and transfinite cardinals, among which the finite ones are just a small class whose properties are to be deduced from the general properties of cardinals. To accomplish this project, logicists resorted immoderately to using formulas without words: "Unfortunately they reached contradictory results, the so-called Cantorian antinomies". Sometimes Poincaré seemed to lay the blame for contradictions on formalism itself; he displayed everywhere a fierce sarcasm against pasigraphy and its putative miraculous effects.

For instance, Poincaré described Burali-Forti's antinomy following to the letter the way its author stated it: two transfinite ordinal numbers $a$ and $b$ may exist such that $a$ is not equal to $b$, nor greater, nor lesser; he then reminded us that Cantor had proven instead that any two transfinite numbers could be compared, just like finite ones. ${ }^{5}$ Poincaré relied on the authority of Jacques Hadamard (1865-1963), who judged Cantor's proof to be perfect and thought that Burali-Forti had no right to consider the class of all ordinals. Poincaré was ironic: how come? He has just defined it, by ${ }^{6}$ $\Omega=T^{\prime}($ No, $\bar{\varepsilon}>)$

Poincaré could not possibly ignore the debate raging in those years about the legitimacy of $\Omega$, but he took no sides; apparently he was merely interested in giving one more example of the harm done by formalism.

Poincaré discussed formalism again when writing about Hilbert's Grundlagen der Geometrie (1899), although here Hilbert, using no symbols from logic, did no more than presenting the philosophy of the axiomatic method in which the meaning of primitive terms such as "point" and "line" was not fixed. However, Poincaré was likely under the effect of the impression given by Hilbert's talk in Heidelberg, and anticipated an interpretation that would be given later (for instance in [39]), which saw in the pure manipulation of symbols the root of a formalist temptation.

However, in Poincaré's opinion, Hilbert's formalism was justifiable in view of its goal: looking for a complete list of as few as possible axioms necessary for geometry. But, just as the art of playing chess does not consist in just moving pieces, so mathematics cannot do without "the

\footnotetext{
${ }_{5}^{5}$ Poincaré rightly gave no weight to what Burali-Forti said to Couturat, as reported by the latter, about his sets being perfectly ordered ones, not Cantor's well-ordered sets [23, p. 304]. Burali-Forti had given a different definition of ordinals, as types of perfectly ordered classes, but then went on confusedly asserting they were the same as Cantor's. It is thought he had not understood the definition. Later, he remarked in [3] that perfectly ordered classes are well ordered, but not the other way around.

${ }^{6}$ The formula means that $\Omega$ is the order type, $\mathrm{T}^{\prime}$, of the class of ordinals (No) taken with the relation of being similar to an initial segment.
} 
instinct, ... the laws of this deep geometry, which are felt, not stated". Poincaré intended to challenge the claim that it was possible to prove mathematical truths without resorting to intuition.

"The principle of complete induction seems to me at once necessary to mathematics and irreducible to logic", together with other similarly irreducible principles. Logicists said that the principle of induction was just a disguised definition of natural numbers. Poincaré impugned the exposition by Couturat and that in [40] (where it is stated that section 3 about natural numbers is due to Russell).

While describing the harm done by pasigraphy, Poincaré also discussed Burali-Forti's 1896 paper [1], which presented a tentative proof that the class of finite classes satisfies the principle of induction.

Poincaré summarized Burali-Forti's work by saying that it gave a proof of the principle of induction that used two postulates: the existence of an infinite class, and one that he transcribed in a symbolic notation:

$$
u \varepsilon \mathrm{K}(\mathrm{K}-\iota \Lambda) . \circlearrowright . u<\smile^{\prime} u \text {. }
$$

In Poincaré's opinion this was false, as, he said, Alfred N. Whitehead (1861-1947) had shown and as everybody would have been able to verify, "if it had been stated in an intelligible language", since it claimed that "the number of combinations which can be formed with several objects is smaller than the number of these objects".

It is not known who helped Poincaré read through that ideogram, but the complete misrepresentation is peculiar. The formal proposition (17 of $\$ 2)$ is just the partition principle, which is indeed incorrect, but for a different reason than the one stated: it is necessary for $u$ to be a class of disjoint classes. In a footnote [1, note 1, p. 46] BuraliForti explained in an intelligible language that the proposition meant that,

every class of non-empty classes is equivalent to a part of the class constituted by the elements constituting its elements. In other words, in Pp17 we exactly express the common notion that 'a class of classes contains at least as many elements as those constituting its elements'.

Poincaré might have just heard that the proposition was wrong, and tried to read it on his own to find the error.

Mario Pieri (1860-1913) was also informed by Couturat, who related the critical remark by Whitehead, the same source quoted by Poincaré, of the untenability of Burali-Forti's formulation. Then Pieri, in [20], corrected Burali-Forti's proof by replacing Burali-Forti's principle with another one which he believed to have the requisites to become an axiom for class theory: "Given an infinite class whose elements are classes themselves, the class constituted by all the elements of these classes is itself infinite". 7

Poincaré likes Pieri's remarks about consistency proofs and his awareness of the "serious difficulties one gets into in order to exclude the notion of integer number", but, in Poincaré's opinion, the principle Pieri intends to substitute for that of Burali-Forti's is no more evident than what has to be proven. There is, once again, a misunderstanding, because Poincaré assumes that Pieri is claiming that the number of possible combinations is finite when the number of elements is finite.

Poincaré [23, p. 311] also discusses a proof of the principle of induction Zermelo wrote him, which relies on the notion of a simple sequence, that is, a well-ordered set in which every element but the first has a predecessor; the terminology is due to Dedekind, as is the proof, which follows the one by Dedekind. However, Poincaré objects that one should prove the existence of an infinite simple sequence first.

As for the attempts to introduce induction as a property defining numbers, the general criticism offered by Poincaré is that definitions have to come together with a proof of consistency, and such a proof can only be given by induction.

One of the arguments Poincare used to counter the logicists is that in their definitions of the numbers, not just in their justification of induction, a petitio principii appears. ${ }^{8} \mathrm{He}$ is ironic about the definition of 1 he finds in [2], given as

$1=\imath \mathrm{T}^{\prime}\{\mathrm{Ko} \frown(u, h) \varepsilon(u \varepsilon \mathrm{Un})\}$,

by the circular presence of "Un" in the right-hand side.

In Burali-Forti's notation, "Un" is the class of singletons; Poincaré remarks that by $1 \varepsilon$ No (" 1 is a number"), which is deduced from the definition, we are told that Un is a number. Poincaré is even more caustic with Couturat, who had explicitly defined "Un" and then avoided using "one" in the definition, but had in return used "two" in his definition of a singleton (if two elements belong to a singleton, then they are equal).

The formula we are talking about, which is formula 26 in [2, p. 161], is transcribed incorrectly; the correct form is $1=\imath T^{\prime}\{K o \frown \overline{(u, h) \varepsilon}(u \varepsilon U n)\}$,

but it is clear that for Poincare the crucial point is the presence of Un.

\footnotetext{
7 "Étant donnée une classe infinie, dont les éléments sont à leur tour des classes, la classe formée par tous les éléments de celles-ci est ellemême infinie" (Pieri's italics).

8 The other one is their use of impredicative definitions; we shall discuss this later on.
} 
When Couturat replies in [6] that in his logical formula the number 2 is not actually used, Poincare retorts that "one cannot speak of $x$ and $y$ without thinking $t w o$ ". More in general, "it is impossible to give a definition without enunciating a sentence, and it is difficult to enunciate a sentence without inserting in it the name of a number, or at least the world 'several,' or at least a word in the plural. And then the slope is slippery, and at each instant one risks falling into a petitio principii” [23, pp. 821-822].

In this case the consensus is that Poincaré is wrong; the misunderstanding was already anticipated by [10], who had shown that words such as "never" (to define zero), "a class with one object" and the like do not require numbers because they can be expressed by means of the logic of quantifiers. Nevertheless, Poincaré claims that it is impossible not to think about numbers.

\section{Proofs of consistency}

Poincaré agreed with Hilbert's idea that "[in mathematics,] existence has a single possible meaning: being free from contradictions", and was similarly aware that it is not always possible to establish it by giving an example that satisfies a definition or a system of axioms.

In [23] the topic is extensively covered, in connection with the axioms for arithmetic the logicists tried to pass off as definitions. Only when the idea that existence is equivalent to consistency, and that the latter has been proven, is embraced, may one accept the idea due to John Stuart Mill (1806-1873) that definitions are axioms in disguise, and conversely that axioms are definitions, since they imply a statement of existence.

In the case of number theory, it was apparent to Hilbert, both in his paper about the concept of number [13] and in his collection of mathematical problems [14], that it was necessary to follow a different road, in order to get an absolute consistency. For this reason, he proposed a different strategy in [15].

Hilbert's 1904 paper was the object of a detailed and merciless criticism by Poincaré. He recognized and appreciated that Hilbert only intended to consider combinations of elements built from bottom up, so to speak; but he sees a double petitio principii, that is, an implicit use of the concept of number starting with the first definition of things as combinations of things, and an explicit use in the proofs by induction, of which he ruthlessly reports every single occurrence.

"The groups formed with this object [the ' 1 ' sign], repeated two, three, or several times..." Ah! Here we are, it is no longer the same; if we introduce the words "two", "three", and above all "more", we introduce the notion of number; and then the definition of finite whole number which we shall find at the end will come too late [23, p. 18].

...How can we show that all these propositions [one may deduce] are identities? Consider a series of consequences deduced from our axioms and halt at a precise step of this series; if at this step we have obtained just identities, we may verify that, by applying any of the operations allowed by logic to these identities, we can only obtain further identities. We may then conclude that we shall never get anything but identities; but arguing like this is doing a complete induction [23, p. 20].

... Hilbert proves next that the first two [equations taken as axioms for the concept of successor] can only lead to homogeneous propositions, i.e., equations with the same number of letters on both sides; $\ldots$ and this is true for the second [equation] too, on condition that the premise is itself a homogeneous equality. Complete induction, once more [23, p. 21].

Hilbert would always bear this criticism by Poincaré in mind, and, sure that it can be surmounted, would take every chance to try to reply to it, in the course of the elaboration of his programme in the 1920s. The awareness of the presence of numerical concepts in his first logic called for a simultaneous development of logic and arithmetic.

In a 1921 seminar in Hamburg, Hilbert said:

But these thoughts about manipulating signs are different from the principle of induction, since they only involve, and rely on, composing and decomposing a sign we may assume as given (For instance, in order to prove $\mathbf{a}+\mathbf{b}=\mathbf{b}+\mathbf{a}$, if $\mathbf{b}>\mathbf{a}$ we decompose $\mathbf{b}$ as $\mathbf{a}$ $+\mathbf{c}$ and move on to a shorter sequence such as $\mathbf{a}+\mathbf{c}$ $=\mathbf{c}+\mathbf{a}$. This procedure) just relies on composing and decomposing numerical signs and is substantially different from that principle that, as principle of complete induction, ... plays such a relevant role in higher arithmetic [16].

While this defence was good for Hilbert's colleagues, not all external observers found it convincing. For instance, Thoralf Skolem (1887-1963), who in 1922 had seen an excerpt from Hilbert's conference, agreed with Poincaré and remarked that one has to be careful to only use properties not depending on, or equivalent to, induction; in this connection, in [37] he criticized Hilbert's reference to the notion that if a symbol appears in a proof there exists a first step in which it appears.

Hilbert's final answer to Poincaré was the one given in 1927 during another seminar in Hamburg:

Poincaré already made in several places statements that conflict with my views; first of all, he denied 
from the outset the possibility of a proof of consistency for the axioms for arithmetic, maintaining that the consistency of the method of mathematical induction could never be proven except through the inductive method itself. But, as my theory shows, when founding arithmetic two distinct methods that proceed recursively come into play, namely, on the one hand, the intuitive composition of integer numbers as numerical signs..., an induction related to content, and, on the other hand, formal induction proper: the latter is based on the induction axiom and through it alone the mathematical variable can begin to play its role in formalism [17].

A year later, while reprinting this work, Hilbert omitted the words "induction related to content" and "formal", leaving the first procedure unnamed and calling the second "induction proper".

At the time, even Weyl, while not far from Hilbert in the debate with Luitzen E. J. Brouwer (1881-1966), recognized in [38] that, although Hilbert emphasized that in metamathematics one only works with concrete objects, he does not handle just $0,0^{\prime}$, and $0^{\prime \prime}$, but any $0^{\prime \prime} \ldots{ }^{\prime}$ :

we may well stress 'concretely given;' on the other hand it is as essential that the arguments about content in proof theory are developed in a hypothetical generality, for any proof, for any numeral.

Weyl did not mean his remark as a criticism, since he recognized that the applications of induction made by Hilbert had the telltale mark of content-oriented thought; nevertheless, he claimed that Poincaré was right in pointing out the difference. Moreover, it was still true that even restricted induction holds because the objects it is applied to are, in the case of numerals, either 1 or a numeral followed by 1 , that is, they are themselves defined inductively. In Weyl's opinion, Poincaré had the merit of pointing out this fundamental fact.

The distinction suggested by Hilbert, which in later years was subjected to many changes and interpretations, can be reasonably summed up as follows: If we require that the properties to which induction may be applied are such that it is always possible to check, in every given case, whether they hold or not and what operations are needed for this check, then in the formal system we are only dealing with propositions that are either free from quantifiers in their prefix or only have universal quantifiers. This corresponds to the induction used in primitive recursive arithmetic, and not to that applied to arbitrary formulas in Peano arithmetic. Of course, after Kurt Gödel's (1906-1978) incompleteness theorem, this controversy between Hilbert and Poincaré's ghost lost all urgency, since not even using actual induction one may prove the consistency of arithmetic.

\section{Impredicative definitions}

Jules Richard (1862-1956) taught mathematics in a high school and was an attentive follower of the advances of mathematics. He had already written a book about philosophy of mathematics [29], when he published a paper [30] in which he described the paradox that now bears his name. The paper begins with a puzzled reference to the contradiction between König's result shown at the 1904 congress, which Richard did not know had been corrected, and the contrasting proof by Zermelo. But in Richard's opinion it was not necessary to advance within the theory of ordinal numbers to find contradictions.

Richard pictures ordering all French sentences lexicographically, deleting those not defining numbers. Then all real numbers between 0 and 1 that can be defined with finitely many words form a well-ordered countable set $E=\{p 1, p 2, \ldots\}$. Richard now considers the number $s$ between 0 and 1 so defined: $s=. a 1 a 2 \ldots$, such that $a n$ is the $n$th decimal digit of $p n$, increased by 1 unless it is 8 or 9 , in which case it is taken to be 1 . Even if $s$ has been defined using finitely many words, $s$ is not in $E$, a contradiction.

Richard does not regard his argument as a true antinomy, but simply as an outward contradiction, so much so that he offers a solution at the same time: defining $s$ requires defining $E$, and the latter actually consists of infinitely many words. If it were possible to define $s$, it would be one of the $p k s$, but in this case, while defining $p k$, one should mention $E$, which was not yet defined. The sentence that seems to define $s$ gets canceled out. ${ }^{9}$

Unlike Richard, Poincaré realized immediately that, if this were the explanation, it would have consequences of no small import, since definitions of that kind were present in all paradoxes and in many of the concepts relevant to the debate about foundations, thus they had to be avoided.

So Poincaré also identified the reason why antinomies appear in the logicists' work; they overcome the contradiction between logic being tautological and mathematics being creative by using impredicative definitions: "Logistic is not sterile: it generates antinomies" [23, p. 315].

The vicious circle lies in defining an entity by referring to a totality of entities to which the entity to be defined belongs. In 1905, in his study about restrictions to be imposed on the comprehension principle, Russell in a general setting called propositional formulas that determine a class "predicative" and those that do not "not predicative". Since for Poincaré the formulas to be excluded were

\footnotetext{
9 In his later paper [31], Richard changed his explanation, maintaining that his definition was to be refused simply because it led to a contradiction.
} 
those entailing a vicious circle, the predicative ones were those without vicious circles.

Impredicative definitions appear outside discussions about antinomies too. For instance, the definition of the set $N$ of natural numbers given in [7] as the intersection of all sets containing an element 1 and closed with respect to taking successors (chains containing 1) is impredicative.

Another example concerns the axiom of choice. Poincaré was willing to accept it:

These axioms [the axiom of choice and Russell's multiplicative axiom] will always be propositions admitted as "self-evident" by some and doubted by others. Everyone will only trust their intuition. Nevertheless, all will agree on one point: the axiom is "self-evident" for finite classes. But if it is not provable for infinite classes, it is certainly not so for finite classes either, the latter ones not being yet distinguished from the former ones at this step of the theory. So it [the axiom of choice] is an a priori synthetic principle without which the "theory of cardinal numbers" would be impossible, both for finite and infinite sets [23, p. 313].

In this evaluation of the axiom of choice we may notice a curious use of the notion of "a priori synthetic" for a proposition somebody admits as evident while others do not, varying from a person to the next one. But it is not logically provable, and without it the theory of cardinal numbers would be impossible.

However, Poincaré refused Zermelo's proof of well ordering, since he saw in it a non-predicative move: Zermelo considered $\gamma$-sets as well-ordered subsets of a given set $A$ in which the minimum of each non-empty subset is singled out by a fixed choice function (covering) $\gamma$; the set $L \gamma$ defined as the union of all $\gamma$-sets is a $\gamma$-set and turns out to be a well ordering for the whole set $A$.

In Poincaré's opinion, every paradox was due to the use of non-predicative definitions. To demonstrate the presence of a vicious circle, Poincare widened the domain of the contradictions he is scrutinizing, including those about definability and even the liar paradox, at the cost of belying his own claim that the contradictions were due to "the Cantorians having forgotten [that actual infinity does not exist]".

In [23] the three proposals to restrict the comprehension principle presented in [33] were discussed in detail: the zigzag theory, the theory of limitation of size, and the no-classes theory. Poincaré's tone was ironical and amused: Russell's proposal of not considering classes as independent entities represented or required a radical reconstruction of logistic, embarrassing the logicians who talked of nothing but classes. "The whole logistic is to be remade".
Russell replied in September $1906^{10}$ with a paper [34] which represents an important step in the development of his thought about antinomies, since on this occasion he accepted Poincaré's suggestion, the disagreement about everything else notwithstanding.

The reply contained several themes, corresponding to the several arguments used by Poincaré. Russell pointed out that the changes required if one were to completely eliminate classes would not be enormous, after all. He compared the situation with that for calculus, which neither assumed nor used infinitesimals anymore, and thus removed some contradictions without modifying any of the formulas. He also discussed a tentative approach to the elimination of classes. Russell then defended the logicists' positions, covering at length the "subject of 'intuition' and the nature of evidence for the truth of propositions in logistic".

However, what's new in this work lies in this sentence:

M. Poincaré holds that these paradoxes all spring from some kind of vicious circle, and in this I agree with him.

Russell agreed both about antinomies and the principle of induction. He took Poincaré's suggestion as stating the thesis according to which "whatever concerns every or some or any member of a class cannot be a member of the class" and then reformulated it in "Peano's language":

Whatever involves an apparent [i.e., bound] variable must not be among the possible values of that variable.

"But [Poincaré] fails to realize the difficulty of avoiding a vicious circle of this sort". The difficulty consists in the necessity of "construct[ing] a [new] theory of expressions containing apparent variables which will yield the viciouscircle principle as an outcome". The vicious-circle principle is not in itself the solution, but rather the result expected from the way in which the theory is constructed. If stated as a directive, it yields in itself a vicious circle.

In 1907 Russell explicitly explained that we cannot say "When I speak of all propositions I mean all except those in which 'all propositions' are mentioned", since in this way we have actually mentioned the propositions in which "all propositions" are mentioned. "It is impossible to avoid mentioning a thing by mentioning that we won't mention it". So, it is necessary "to construct our logic without mentioning such things as 'all propositions' or 'all properties,' and without even having to say that we are excluding such things. The exclusion must result naturally and inevitably from our positive doctrines, which must

\footnotetext{
${ }^{10}$ It has to be noted that exchanges happened in real time, thanks to direct communication and the absence of a publication backlog.
} 
make it plain that 'all propositions' and 'all properties' are meaningless phrases". This doctrine, elaborated in [35], is the ramified theory of types and is adopted in 1910-13 Principia mathematica. However, Russell had already considered it even earlier, in 1903 in [29], without choosing it as a solution.

In 1909 Poincaré showed his appreciation for the acknowledgment of the vicious-circle principle by Russell, even if he now stated it in a slightly different way, emphasizing the fact that sets had to receive a stable definition, one not modified by the introduction of later elements: infinite sets are generated progressively, ${ }^{11}$ and when new elements are added the relation between the set and the elements already in it is modified; some of them might even have to be removed. This happens, for instance, when the elements are defined by a sentence of finite length; as new elements are added and the set is modified, the meaning of the sentence changes, elements not defined by it become so, while elements previously defined by it aren't anymore.

He named the definitions for which this does not happen "predicative", and he did not find them explicitly in Russell's formulation, which on the other hand he read somewhat inaccurately, confusing types and orders. In his summary he claimed that a proposition is not always of the same order regardless of $x$, since its order depends on $x$; the function will be called "predicative" if it is of order $k+1$ when $x$ is of order $k$. Poincaré complained that Russell gave no examples and that he did not properly explain the purpose of the axiom of reducibility, which should be used in the proof of the principle of induction. Poincaré would have appreciated this, since he suspected it to be just another form of the same principle; the attempts at proving the principle of induction reminded him of the attempts at proving Euclid's fifth postulate by assuming one of its consequences as an obvious truth.

As for the hierarchy of orders, Poincaré remarked that Russell did not make it clear whether, apart form propositions of order $n$, for every $n$, which can be defined in a natural way, there exist propositions of order $\alpha$ too, where $\alpha$ is a transfinite ordinal number, in particular $\omega$. If this is so, one should explain what these propositions might be; otherwise, we are supposed to already be able to distinguish between the finite and infinite cases.

Poincaré tried to propose an application of his own of the axiom of infinity: the definition of natural numbers as

\footnotetext{
11 In the conclusions of [23, pp. 316-7], he goes so far as to state that actual infinity does not exist and in [28, p. 150] he claims that pragmatists, among which he places himself, only accept entities definable with finitely many words, in contrast to the Cantorians. In [25, pp. 132-3], he claims more resolutely that an objects exists only when it can be defined with finitely many words, since in order to exist it has to be conceivable.
}

those belonging to all recurrent classes is meaningless if we do not state the orders of the recurrent classes; then, there are numbers of different orders; but if a number is of order $n$, it is of order $n-1$ too, so we get an increasingly restrictive sequence of sets. We could say the numbers belonging to all orders $n$ to be of order $\omega$, so obtaining an operative definition.

In [36], Russell took note of the non-contentious tone of Poincaré, and since the latter asked for further clarifications, he seized the opportunity to discuss in more detail the motivations and philosophical interpretations of his system, which he could not cover in the paper due to appear in a mathematical journal. The exposition is indeed longer and more detailed than that in [35], but Russell did not answer any of Poincaré's questions.

To conclude the analysis of [23], we must recall an important remark about the Cantor-Bernstein theorem, or the equivalence theorem, as it was also called, on pages 27-29 and 314-315. ${ }^{12}$ This is also connected to the issues about impredicativity. By the equivalence theorem, if there is an injection of $A$ in $B$ and an injection of $B$ in $A$, then there is a bijection between $A$ and $B$. This is crucial to give a foundation to cardinality theory, since it allows us to prove that if $\operatorname{card}(A) \leq \operatorname{card}(B)$ and $\operatorname{card}(B) \leq \operatorname{card}(A)$, then $\operatorname{card}(A)=\operatorname{card}(B)$; actually, it is equivalent to the antisymmetry of $\leq$.

The first proof, due to Felix Bernstein (1878-1956) in 1897, reduced to proving that if $A, B$, and $C$ are three disjoint sets, and there is a bijection $g$ between $A \cup B \cup C$ and $C$, then there is a bijection between $A \cup B \cup C$ and $B \cup C$.

In order to prove the existence of the latter, we use a decomposition based upon the bijection $g$, illustrated by the following diagram, where $B_{i_{+1}}$ is the image of $B_{i}$ in the given bijection $g$ between $A \cup B \cup C$ and $C$, and analogously $A_{i_{+1}}$ is the image of $A_{i}$; a remainder $R$ is possible:

\begin{tabular}{|l|l|l|l|l|l|l|l|}
\hline$A$ & $B$ & \multicolumn{5}{|c|}{$C$} \\
\hline$A$ & $B$ & $A_{1}$ & $B_{1}$ & \multicolumn{2}{|c}{} \\
\hline$A$ & $B$ & $A_{1}$ & $B_{1}$ & $A_{2}$ & $B_{2}$ & & $R$ \\
\hline
\end{tabular}

The desired bijection is defined to be the identity on $R$, on $B$, and the $B_{i} \mathrm{~s}$, and equal to $g$ on the set

$Z(A)=\cup\left\{g^{n}(A): n \in N\right\}$

\footnotetext{
${ }^{12}$ Unfortunately this remark was removed in the condensed version in [24].
} 
where $g^{n}(A)=A_{n}$ and $A_{0}=A$ : it is easy to verify that the map has the required properties.

The use of natural numbers in Bernstein's proof was seen by Poincaré as a confutation of the logical precedence of the notion of cardinality over that of number, and a confirmation of the necessity of numerical intuition when constructing a cardinality theory. The proof could only be accepted "if we consider the principle of induction to be a synthetic proposition, rather than a definition, since such a definition would be 'non predicative'" [23, p. 314].

\section{Peano's and Zermelo's replies}

Poincaré's refusal of impredicative definitions was criticized by Peano in [19] and by Zermelo in [42] and [43].

In his "Additione" to the Cantor-Bernstein theorem, Peano took into consideration both the axiom of choice and the antinomies, the one by Burali-Forti and Russell and the one by Richard. In [18], Peano had proposed a proof of the equivalence theorem based upon the theory of Dedekind chains. An analogous proof was mailed by Zermelo to Poincaré, who related it in [23, pp. 314-315].

Peano kept Bernstein's general layout, substituting the definition of $Z(A)$ with another which, translating from Peano's formalism, can be written:

$Z(A)=\cap\{v: A \subseteq v \cdot g(v) \subseteq v\}$

or, recalling the notation above,

$Z(A)=\cap\{v: A \cup B \cup C: A \subseteq v \cdot g(v) \subseteq v\}$,

where $g(v)=\{g(x) \mid x \in v\}$.

In Peano's words (in Latino sine flexione), $Z(A)$ is the parte comune ad classes $\mathrm{v}$ tale que functione $\mathrm{g}$ transforma $\mathrm{v}$ in parte de $\mathrm{v}$, et que contine A (part common to classes $v$ such that the function $g$ transforms $v$ into a part of $v$ and that it contains $A$ ).

In the same "Additione", Peano also discussed the concept of predicativity. In Peano's opinion, Poincaré was wrong when he denounced a vicious circle in Richard's argument; there was no vicious circle, but just a reference to everyday language, ubi es puncto debile in argumento (where the weak point of the argument is).

Mathematics could not exist if we were to accept the restrictions urged by Poincaré who, in Peano's opinion, would even consider the definitions of $b-a$, of the least common multiple of two numbers, or of $\sqrt{ } 2$, to be impredicative. On the other hand, if in Richard's argument by "notion $E$ " we mean something equivalent to $E$, we would find impredicativity in every definition, since the definiens has to be equivalent to what is being defined.

So, Poincaré's demand was not only excessive, but it was not even sufficient. Now Peano was lecturing Poincaré about definitions. In his Formulario Peano had already made clear - in his opinion, better than Poincaré did - the constraints on definitions. Every definition has to be of the form

$x=$ an expression only containing signs preceding $x$ in the orderly and systematic construction of a theory.

Poincaré, on the other hand, would have demanded that every definition be of the form

$$
\begin{aligned}
& x=\text { an expression only containing signs different } \\
& \text { from } x \text {, }
\end{aligned}
$$

without excluding that in the expression used in the definition other symbols conceptually successive to $x$ appear.

In [42] Zermelo addressed a section titled "Objection about non-predicative definition" exclusively to Poincaré:

The point of view defended here, that we deal with a productive science ultimately relying on intuition, was recently advocated by Poincaré in a series of essays, against Peano's 'logistic'.

Poincaré gave the axiom of choice, which he saw as an unprovable but indispensable axiom, its due. Nevertheless,

his attack is so encompassing - since his adversaries deal mainly with set theory - that he identifies the whole of Cantor's theory, this original creation by a specifically mathematical thought and the intuition of a genius, with the logistic he fights against, denying it every right to exist, regardless of its positive results, only on the strength of the as-yet-unresolved antinomies. $^{13}$

If Poincaré had only intended to exact the presence of $a$ priori synthetic propositions in the foundations of arithmetic, including especially the principle of induction, in Zermelo's opinion it would have been sufficient to attribute this characteristic to the fundamental propositions on which the set-theoretic proofs of this principle relied. Even the supporters of set theory would have accepted it, the distinction between "analytic" and "synthetic" basically being a purely philosophical one, with no consequences for mathematics. On the contrary, Poincaré ventured to fight those proofs wielding formal logic as his weapon, on a field where his adversaries had the advantage.

Zermelo recalled Poincaré's refusal of the theory of Dedekind chains and of the definition of $L_{\gamma}$ in his 1904 proof. But the proofs with this logical form, Zermelo remarked, were not confined to set theory; the same kind of

\footnotetext{
${ }^{13}$ See [23, p. 316]: "There is no actual infinity; Cantorians forgot this, and contradicted themselves" (note by Zermelo).
} 
proofs was found in analysis, every time that the maximum or the minimum of a previously defined "complete" (i.e., closed) set $Z$ was used for further inferences. For instance, it occurred in the well-known proof by Cauchy of the fundamental theorem of algebra, and up to this time nobody had viewed it as illogical.

On the other hand, it is precisely the form of a so-called predicative definition which contains something circular; in fact, unless we already possess the notion, we have no way to know which objects will be in future determined by it and which ones excluded. Of course, it is true that the question of whether an arbitrary given object falls within a definition has to be decidable independently of the notion yet to be defined, by an objective criterion. But when such a criterion is given, as it is in fact in all cases pointed out in my proofs, nothing can prevent that some of the objects subsumed under the definition have a further special relation with the notion so defined, and are in this way determined by, or distinct from, the remaining ones - for instance, an intersection or a minimum. After all, an object is not created via such a "determination"; on the contrary, every object can be determined in a large variety of ways, and these different determinations do not yield identical notions but only equivalent ones, that is, notions with the same extension.

Apparently Poincaré disregarded the existence of equivalent notions, as Peano accused him; Zermelo related and agreed with Peano's objections to Poincaré [43]. ${ }^{14}$

Zermelo remarked about the equivalence theorem:

The proof of the equivalence theorem given here in sections 25 and 27 (first published by Poincaré [23, pp. 314-315] on the basis of a letter I wrote him in January 1906) relies only on the theory of Dedekind chains and, unlike previous proofs by Schröder and F. Bernstein, as well as the recent proof by J. König, it avoids any reference to ordered sequences of order $\omega$ or to the principle of mathematical induction. At about the same time, Peano published a quite similar proof; the paper containing the proof also contains a discussion of the objection directed by Poincaré against my proof. $^{15}$

Poincaré was not responsive to this criticism. In [23, pp. 314-315], he concisely but correctly summarized

\footnotetext{
${ }^{14}$ Except for one remark, discussed in next footnote.

15 In a footnote, Zermelo complains about a breach of manners by Peano who in [19] wrote as if Poincaré were referring to Peano's proof, without making clear that the proof was actually Zermelo's.
}

Zermelo's proof he had received by mail, together with his reply ${ }^{16}$ :

The error is one more the same [as in Richard's antinomy]: $Z(A)$ is contained in all sets [ $v$ ]; to avoid a vicious circle, we have to understand this as meaning "all sets whose definition does not involve the notion of $Z(A)$ ". This excludes the set $A * g(Z(A))$, which depends on $Z(A) .{ }^{17}$ The definition of the set $Z(A)$ is not predicative [23, p. 315].

Poincaré did not believe that mathematics would be mutilated by it, since he trusted that many impredicative proofs could be reformulated in an acceptable way. For instance, in [26] and [27] he suggested a way to avoid the impredicativity in the proof of the fundamental theorem of algebra, which Zermelo had mentioned as being one of the results to be lost to predicative constraints. Zermelo managed to have the final say, by replying in an appendix to [43] with a rhetorical gambit that turns a sentence by Poincaré into an acceptance of Zermelo's definitions ${ }^{18}$ :

More in general, if we consider a set $E$ of positive real numbers [of sets $M_{1}$ ], it can be proven that this set admits an infimum $e$ [a common subset $M_{0}$ ]; this infimum [common subset] is defined after the set $E$; and we are not begging the question, since $e\left[M_{0}\right]$ does not in general belong to $E$. In some particular cases, it might happen that $e\left[M_{0}\right]$ belongs to $E$. In these particular cases, we are all the same not begging the question, because $e\left[M_{0}\right]$ does not belong to $E$ as a consequence of its definition, but of a proof subsequent to both the definition of $E$ and of $e\left[M_{0}\right]$ [26, p. 199; 44, p. 193].

The conversation progressed no further, and everyone remained of his own opinion.

Translated from the Italian by Daniele A. Gewurz

\section{References}

1. Burali-Forti, C.: Le classi finite. Atti R. Acc. Scienze Torino 32, 34-52 (1896)

2. Burali-Forti, C.: Una questione sui numeri transfiniti. Rendiconti del Circolo Matematico di Palermo. 11, 154-164 (1897); English translation in J. van Heijenoort, From Frege to Gödel. Harvard University Press, Cambridge, 1967, pp. 104-111

3. Burali-Forti, C.: Sulle classi bene ordinate. Rendiconti del Circolo Matematico di Palermo. 11, 260 (1897); English transl. in J.

\footnotetext{
${ }^{16}$ In the quote that follows we maintain the notation given in the above sketch of the proof.

17 And which in Zermelo's proofs is shown to be equal to $Z(A)$.

18 The quote that follows is by Poincaré; we give in brackets the words Zermelo substitutes for Poincaré's.
} 
van Heijenoort, From Frege to Gödel, Harvard University Press, Cambridge, 1967, pp. 104-112

4. Couturat, L.: Les principes des mathématiques. Revue de Métaphysique et de Morale. 12, 19-50, 211-240, 664-698, 810-844 (1904)

5. Couturat, L.: La philosophie des mathématiques de Kant. Revue de Métaphysique et de Morale 12, 321-383 (1904)

6. Couturat, L.: Pour la logistique (Réponse à M. Poincaré). Revue de Métaphysique et de Morale 14, 208-250 (1906)

7. Dedekind, R.: Was sind und was sollen die Zahlen? Vieweg, Braunschweig (1888); English translation What are numbers and what should they be?, in From Kant to Hilbert, 2 vols., Ewald, W.B. (eds.) Oxford University Press, Oxford, pp. 787-832 (1996)

8. Detlefsen, M.: Poincaré against the logicians. Synthese 90(3), 349-378 (1992)

9. Ferreirós, J.: Labyrinth of thought. Birkhäuser, New York (2007)

10. Frege, G.: Die Grundlagen der Arithmetik. Koebner, Breslau (1884)

11. Frege, G.: Briefwechsel mit D. Hilbert, E. Husserl, B. Russell sowie ausgewählte Einzelbriefe Freges. In: Gabriel, G., Kambartel, F., Thiel, C. (eds.) Felix Meiner Verlag, Hamburg (1980)

12. Goldfarb, W.: Poincaré against the Logicists. In: Aspray, W., Kitcher, Ph. (eds.) History and philosophy of modern mathematics. The University of Minnesota Press, Minneapolis, pp. 62-81 (1985)

13. Hilbert, D.: Über den Zahlbegriff. Jahresbericht der DMV 8, 180-184 (1900)

14. Hilbert, D.: Mathematische Probleme, Nachrichten von der Königlichen Gesellschaft der Wissenschaften zu Göttingen. pp. 253-297 (1900)

15. Hilbert, D.: Über die Grundlagen der Logik und der Arithmetik. Verhandlungen des Dritten Internationalen Mathematiker-Kongresses in Heidelberg, vom 8. bis 17. August 1904. Teubner, Leipzig, pp. 174-185 (1905)

16. Hilbert, D.: Neubegründung der Mathematik. Erste Mitteilung. Abhandlungen aus dem mathematischen Seminar der Hamburgischen Universität. 1, 157-177 (1922)

17. Hilbert, D.: Die Grundlagen der Mathematik. Abhandlungen aus dem mathematischen Seminar der Hamburgischen Universität. 6, 65-85 (1928)

18. Peano, G.: Super theorema de Cantor-Bernstein. Rendiconti del Circolo Matematico di Palermo. 21, 360-366 (1906); also in Revista de matematica, vol. viii, n. 5, 1902-1906, pp. 136-143, reprinted in G. Peano, Opere Scelte, Roma, Cremonese, 1957, vol. I, pp. 337-344

19. Peano, G.: Additione a Super Theorema de Cantor-Bernstein. Revista de Mathematica. viii(5), 143-157 (1902-6)

20. Pieri, M.: Sur la compatibilité des axiomes de l'arithmétique. Revue de Métaphysique et de Morale. 13, 196-207 (1906), reprinted in M. Pieri, Opere scelte, Cremonese, Roma, 1980, pp. $377-388$

21. Poincaré, H.: Du rôle de l'intuition et de la logique en mathématiques. In: E. Duporcq (ed.) Compte rendu du deuxième congrès international des mathématiciens tenu à Paris du 6 au 12 août 1900. Gauthiers-Villars, Paris, pp. 115-130 (1902)

22. Poincaré, H.: La science et l'hypothèse. Flammarion, Paris, 1902; English translation Science and Hypothesis, Greenstreet W.J., translation, Walter Scott Publishing, New York (1905)

23. Poincaré, H.: Les mathématiques et la logique. Revue de Métaphysique et de Morale 13 (1905), pp. 815-835, 14 (1906), pp. 17-34 and 294-317, abridged in Poincaré, H., Science et méthode, Flammarion, Paris, 1908, pp. 152-171, 172-191 and 192-214, in three chapters. "Les mathématiques et la logique", "Les logiques nouvelles", "Les derniers efforts des logisticiens", approximatively corresponding to the three parts of the original article. H. Poincaré, Science et méthode, Flammarion, Paris, 1908

24. Poincaré, H.: La logique de l'infini. Revue de Métaphysique et de Morale. 17, 451-482 (1909), also in H. Poincaré, Dernières pensées, Flammarion, Paris, 1913, pp. 101-141

25. Poincaré, H.: Réflexions sur les deux notes précédentes. Acta Mathematica 22, 195-200 (1909)

26. Poincaré, H.: Über transfinite Zahlen, a talk given in Göttingen, published in H. Poincaré, Oeuvres, Gauthier-Villars, Paris, 1921-56, vol. 11, pp. 120-124; English translation in Ewald, W.B. (ed.), From Kant to Hilbert, 2 vols., vol. 2, Oxford University Press, Oxford, pp. 1071-1074 (1996)

27. Poincaré, H.: Les mathématiques et la logique. In: Poincaré, H. (ed.) Dernières pensées. Flammarion, Paris. pp. 143-162 (1913)

28. Richard, J.: Sur la philosophie des mathématiques. GauthierVillars, Paris (1903)

29. Richard, J.: Les principes des mathématiques et le problème des ensembles. Revue générale des sciences pures et appliquées 16, 541-542 (1905)

30. Richard, J.: Sur un paradoxe de la théorie des ensembles et sur l'axiome Zermelo. L'Enseignement Mathématique 9, 94-98 (1907)

31. Russell, B. (1903) The principles of mathematics, vol. I. Cambridge University Press, Cambridge

32. Russell, B.: On Some Difficulties in the Theory of Transfinite Numbers and Order Types. Proceedings of the London Mathematical Society. 4(2), 29-53 (1906), reprinted in B. Russell, Essays in Analysis, D. Lackey, ed. George Allen \& Unwin, London, 1973, pp. 134-164

33. Russell, B.: Les paradoxes de la logique. Revue de Métaphysique et de Morale. 14, 627-650 (1906) later published in English under the title of the original manuscript "On 'Insolubilia' and their Solution by Symbolic Logic”, in B. Russell, Essays in Analysis, D. Lackey (ed.) George Allen \& Unwin, London, 1973, pp. 190-214

34. Russell, B.: Mathematical Logic as based on the Theory of Types. Am. J. Math. 30, 222-62 (1908), reprinted in B. Russell, Essays in Analysis, Lackey, D. (ed.) George Allen \& Unwin, London, 1973, pp. 57-102 and in J. van Heijenoort, From Frege to Gödel, Harvard University Press, Cambridge, pp. 152-182 (1967)

35. Russell, B.: La Théorie des types logiques. Revue de Métaphysique et de Morale. 18, 263-301 (1910), reprinted in English in B. Russell, Essays in Analysis, D. Lackey, ed. George Allen \& Unwin, London, pp. 215-252 (1973)

36. Skolem, T.: Einige Bemerkungen zur axiomatischen Begründung der Mengenlehre. In Mathematiker Kongressen i Helsingfors den 3-7 Juli 1922, Akademiska Bokhandeln, Helsinki, 1923, pp. 217-232; English translation in J. van Heijenoort, From Frege to Gödel, Harvard University Press, Cambridge, pp. 291-301 (1967)

37. Weyl, H.: Das Kontinuum. Kritische Untersuchgungen über die Grundlagen der Analysis. Veit, Leipzig (1918)

38. Weyl, H.: Diskussionsbemerkungen zu dem zweiten Hilbertschen Vortrag über die Grundlagen der Mathematik. Abhandlungen aus dem Mathematischen Seminar der Hamburgischen Universität. 6, 86-88 (1928); English translation in van Heijenoort J., From 
Frege to Gödel, Harvard University Press, Cambridge, pp. 480-484 (1967)

39. Whitehead, A.N.: On cardinal numbers. Am. J. Math. 24, 367-394 (1902)

40. Zermelo, E.: Beweis, dass jede Menge wohlgeordnet werden kann (Aus einem an Herrn Hilbert gerichteten Briefe). Mathematische Annalen. 59, 514-516 (1904); English translation in van Heijenoort, J., From Frege to Gödel, Harvard University Press, Cambridge, pp. 139-141 (1967)

41. Zermelo, E.: Neuer Beweis für die Möglichkeit einer Wohlordnung. Mathematische Annalen. 65, 107-128 (1908); English translation in van Heijenoort, J., From Frege to Gödel, Harvard University Press, Cambridge, pp. 183-198 (1967)

42. Zermelo, E.: Untersuchungen über die Grundlagen der Mengenlehre I. Mathematische Annalen. 65, 261-281 (1908); English translation in van Heijenoort, J., From Frege to Gödel. Harvard University Press, Cambridge, pp. 199-215 (1967)

43. Zermelo, E.: Sur les ensembles finis et le principe de l'induction. Acta Mathematica 32, 185-193 (1909)

\section{Author Biography}

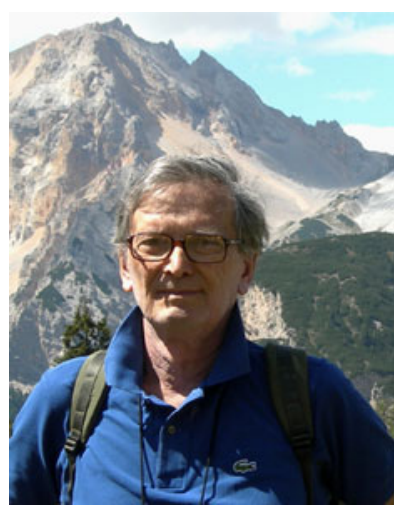

Gabriele Lolli received his degree in mathematics from the University of Torino. His first interests were in axiomatic set theory, later the applications of logic to computer science and Artificial Intelligence; most recently, history and philosophy of logic and mathematics, with special reference to mathematical proof and the origins of set theory. He is currently Professor of the Philosophy of Mathematics at the Scuola Normale Superiore di Pisa. 